\title{
X-Ray Crystalline Undulator Radiation in Water Window
}

\author{
H. Gevorgyan \\ A.I. Alikhanyan National Scientific Laboratory, Yerevan, Armenia \\ E-mail: hayk.gevorgyan@aanl.am
}

Received 19 April 2021

\begin{abstract}
The problem of radiation produced by the bunch of relativistic positrons, channeled into the crystalline undulator, is considered, taking into account the polarization of a crystal medium. If a bunch energy is above the threshold energy, then the radiation is generated in the limited frequency range, due to the medium polarization. Both soft and hard boundary photons are emitted at a zero angle. When the ratio of the bunch energy to the threshold energy approaches unity from above, the peak of the bunch radiation spectrum is near the resonant frequency and can fall into the soft X-ray frequency range due a certain choice of the parameters of the crystalline undulator. In that case, it is necessary to use a low energy relativistic bunch. Method is effective because a fairly dense beam of soft X-ray photons is generated. It is shown the possibility of obtaining monochromatic, intense, directed photon beams in the water window region, important for medical and biological applications.
\end{abstract}

\section{https://doi.org/10.52853/18291171-2021.14.2-105}

Keywords: tunable radiation, crystalline undulator, positron bunch, medium polarization, water window, channeling

\section{Introduction}

Let consider crystalline undulator $(\mathrm{CU})$ with the sinusoidal form of the bending curvature, i.e. the trajectory of the oscillating positrons, channeled between the crystallographic planes, is on average sinusoidal too. Therefore, in addition to the channeling radiation, crystalline undulator radiation (CUR) is also generated. An idea of using an oscillating relativistic bunch of charged particles to form the radiation, belongs to Ginzburg [1]. The problem of X-ray undulator radiation was first considered by Korkhmazyan [2]. Gamma radiation of the particles, channeled into the crystal, was investigated by Kumakhov [3]. Channeling phenomenon explained by Lindhard [4]. Characteristics of undulator radiation in a dispersive medium were revealed in [5]. Various types and cases of spontaneous electromagnetic radiation are discussed in [6]. Generation of radiation, using crystalline undulator, was proposed in [7]. The spectral distributions of radiation, formed in CU, with [8] and without [9] taking into account the medium polarization, were investigated. Consideration of medium polarization, like in the case of bremsstrahlung radiation [10], significantly affects the characteristics and generation conditions of radiation. The upper threshold appears for the crystal bending degree, and is lower than the threshold [8] at which, due to presence of centrifugal force [11], dechanneling of a charged particle occurs. Modulated bunch coherent radiation at a resonant frequency, formed in a dispersive medium of $\mathrm{CU}$, was obtained [12]. Experimental results on CUR were obtained in [13, 14], using positron bunches with energies $0.3 \div 10 \mathrm{GeV}$. Papers on undulator radiation [15-18] have predicted that, at certain particle energies, the influence of medium polarization will lead to the narrowing of the radiation spectrum in the $\mathrm{X}$-ray region. Investigated narrowing effect of the radiation spectral-angular distribution can be 
important for the generation of stimulated radiation [9], as was shown for gas-filled free-electron lasers [19].

Article shows the possibility to obtain a sufficiently intense monochromatic directed beam of photons within the energies $0.28 \div 0.54 \mathrm{keV}$ or within the wavelengths $2.3 \div 4.4 \mathrm{~nm}$. Absorption lengths of these biologically important water window soft X-ray photons are more and less in water and in carbon accordingly, than for the photons of other regions.

Study is based on the effect of narrowing of the frequency-angular spectrum, which takes place when the parameters of $\mathrm{CU}$ are chosen so that the threshold energy for the formation of radiation in dispersive medium is equal to the positron bunch energy. Then zero angle radiated photons' energy is defined only by the parameters of CU and a positron bunch with low relativistic energy is used. Choice of CU parameters defines the energy of emitted soft photons when the bunch energy exceeds the threshold energy [20]. Such photon beams can be generated also using the other methods [21, 22].

\section{Conservation of Planar Channeling of Positrons in CU}

Relativistic positrons, falling parallel into the crystallographic planes of a crystal, interact with many atoms of a crystal simultaneously. Thus, positrons move between the crystallographic planes in the average potential field of the atoms, for which the harmonic law is a good approximation [6]. Thereby, positrons are oscillating according to the harmonic law and with the frequency depending on the energy. The phenomenon of channeling is conserved at falling angles smaller than the Lindhard critical angle $\Theta_{L}=\sqrt{2 v / \gamma}$, where $v$ and $\gamma$ are the potential well depth and the positron energy respectively, in the units of the rest energy of a positron $m c^{2}$. At the large falling angles, the transverse kinetic energy becomes more than the potential well depth, and the movement becomes infinite.

Let consider that longitudinal section of CU crystallographic planes has the sinusoidal form. Then channeling phenomenon will take place for the all positrons with the normal falling vector, and if the maximal angle of the crystal bending is smaller than the Lindhard angle $\Theta_{\max }<\Theta_{L}$. CU parameter is defined as $q_{c u}=\Theta_{\max } \gamma$. Definition of the channeling parameter $q_{c h}=\Theta_{L} \gamma$ laeds to the sufficient condition for existence of $\mathrm{CU}-$ the channeling conservation condition in $\mathrm{CU}-q_{c u}<q_{c h}$. For sinusoidal CU, we have $\Theta_{\max }=2 \pi A / l$, where $A$ and $l$ are the amplitude and the space period of $\mathrm{CU}$ accordingly, therefore, the limiting condition for $\mathrm{CU}$ parameter is $2 \pi A / l<\sqrt{2 \nu / \gamma}$.

\section{Photon Number Spectral Distribution}

Radiation wave-vector depends on the dielectric permittivity of the medium. Since generation of the radiation occurs in the dispersive medium of $\mathrm{CU}$, the permittivity is given by the following formula

$$
\varepsilon(\omega)=1-\left(\frac{\omega_{p}}{\omega}\right)^{2},
$$

where $\omega \gg \omega_{p}, \omega$ is the radiation frequency, and $\omega_{p}$ is the medium plasma frequency. Ignoring that the positron velocity $V$ is changed at the radiation process, and supposing that it moves with the average longitudinal constant velocity $V_{\|}$in CU (dipole approximation), for the number frequencyangular distribution of radiated photons turns out the following formula [5]

$$
\frac{d^{3} N_{p h}}{d \omega d(\theta)^{2} d \varphi}=\frac{\alpha n^{2} \omega \sqrt{\varepsilon}}{2 \Omega^{2}} \sum_{k=1}^{\infty} F\left(z_{k}\right) J_{k}^{2}(a)\left(\beta_{\|}^{2} \sin ^{2} \theta-\frac{k}{a} \beta_{\|} \beta_{\perp} \sin 2 \theta \sin \varphi+\frac{k^{2}}{a^{2}} \beta_{\perp}^{2}\left(1-\sin ^{2} \theta \sin ^{2} \varphi\right)\right),
$$




$$
F\left(z_{k}\right)=\frac{\sin ^{2} z_{k}}{z_{k}^{2}}, \quad z_{k}=\pi n\left(\frac{\omega}{\Omega}\left(1-\beta_{\|} \sqrt{\varepsilon} \cos \theta\right)-k\right), \quad a=\frac{\omega}{\Omega} \beta_{\perp} \sqrt{\varepsilon} \sin \theta \sin \varphi,
$$

where $\alpha=1 / 137$ is the fine structure constant, $\Omega=2 \pi c / l$ is the oscillation frequency of a positron, $l$ and $n$ are the space period and the period number of CU accordingly, $c$ is the light velocity in vacuum, $\beta_{\|}=V_{\|} / c, \beta_{\perp}=V_{\perp} / c \approx \Theta_{\max }, V_{\perp}$ is the maximum value of the positron transverse velocity, $\theta$ and $\varphi$ are the polar and azimuthal angles of radiation respectively, $k$ is the harmonic number, $J_{k}$ is the Bessel function of the order of $k$.

Objective of the article is to obtain directed radiation. At a zero angle, only the first harmonic of the radiation is formed. For the small parameter $a$ with the accuracy of $a^{2} / 4$ in the formula (2), only the first harmonic retains. The photon number frequency-angular distribution for the first harmonic radiation, taking into account that $\varepsilon \approx 1$, has the following form

$$
\begin{gathered}
\frac{d^{3} N_{p h}}{d \omega d(\theta)^{2} d \varphi}=\frac{\alpha \omega}{2}\left(\frac{n \beta_{\perp}}{2 \Omega}\right)^{2}\left(\cos ^{2} \varphi+\sin ^{2} \varphi\left(1-\frac{\omega}{\Omega} \theta^{2}\right)^{2}\right) F(z), \\
F(z)=\frac{\sin ^{2} z}{z^{2}}, \quad z=\pi n\left(1-\frac{\omega}{\Omega}\left(1-\beta_{\|} \sqrt{\varepsilon} \cos \theta\right)\right),
\end{gathered}
$$

where $q_{c u}=\gamma \beta_{\perp}$ is the CU parameter. After integration over $\varphi$ one gets

$$
\frac{d^{2} N_{p h}}{d \omega d(\theta)^{2}}=\frac{\pi \alpha \omega}{2}\left(\frac{n \beta_{\perp}}{2 \Omega}\right)^{2}\left(1+\left(1-\frac{\omega}{\Omega} \theta^{2}\right)^{2}\right) F(z) .
$$

Function $F(z)$ takes it's maximum value equal to one at $z=0$. Since expression $\beta^{2}=\beta_{\|}^{2}+\beta_{\perp}^{2} / 2$ exists due to the sinusoidal nature of the transverse motion of a positron, then one gets $\beta^{2}=1-\gamma^{-2}$ and $\beta_{\|}^{2}=1-\gamma_{\|}^{-2}$. Hence, the longitudinal Lorentz factor is $\gamma_{\|}=\gamma / \sqrt{Q}$, where $Q=1+q^{2} / 2$. In the laboratory frame, due to the Doppler effect, the frequency of the radiation of a relativistic positron is much more than $\mathrm{CU}$ frequency $\omega \gg \Omega$. As a result, energy and impulse conservation law at $z=0$ is satisfied at the small angles of radiation $\theta \ll 1$

$$
\begin{gathered}
z(\omega, \theta)=\frac{\pi n \omega}{2 \Omega}\left(\theta^{2}-\phi(\omega)\right), \phi(\omega)=\omega_{p}^{2}\left(\frac{1}{\omega_{\min }}-\frac{1}{\omega}\right)\left(\frac{1}{\omega}-\frac{1}{\omega_{\max }}\right), \\
\omega_{\min } \leq \omega \leq \omega_{\max }, \\
\omega_{\max }=\frac{\Omega \gamma^{2}}{Q}\left(1 \mp \sqrt{1-\left(\frac{\gamma_{t h}}{\gamma}\right)^{2}}\right), \quad \gamma_{t h}=\frac{\omega_{p}}{\Omega} \sqrt{Q},
\end{gathered}
$$

where $\gamma_{t h}$ is the energy threshold for the generation of radiation in a dispersive medium. Phenomenon of in-plane channeling of positrons in CU persists if the maximum positron angle of the bunch, relative to the channel planes, is less than the Lindhard angle. Note that the number of emitted photons is proportional to $\beta_{\perp}^{2}=q_{c u}^{2} / \gamma^{2}$. With decreasing $\gamma$, the square of the angle $\beta_{\perp}$ increases quadratically and $\Theta_{L}^{2}=2 v / \gamma$ increases linearly. Consequently, taking into account the condition $\beta_{\perp}<\Theta_{L}$, the smaller Lorentz factor $\gamma$, the greater the number of emitted photons. Minimum value of $\gamma$ is limited by the threshold value $\gamma_{t h}$. At minimum value of Lorentz factor $\gamma=\gamma_{t h}$, photons are emitted at a resonant frequency $\omega_{r}=\omega_{p}^{2} / \Omega$. Note that the argument of the nonnegative function $F(z)$ changes within $[0,1]$ when the absolute increment of the argument is $|\Delta z=\pi|$, i.e. the area bounded by the function $F(z)$ is equal $\pi$. At the resonant frequency, $\phi\left(\omega_{r}\right)=0$ with the angular spread $\Delta \theta^{2}=2 \Omega /\left(n \omega_{r}\right)$. Neglecting the term $\omega \theta^{2} / \Omega \leq \omega_{\Delta} \theta^{2} / \Omega=2 / n$ 
$(n \gg 1)$ in (4), and as a result of integration (4) over the variable $\theta^{2}$, for the frequency distribution, one obtains

$$
\frac{d N_{p h}(\omega)}{d \omega}=\frac{\pi \alpha n}{2 \Omega} \beta_{\perp}^{2}
$$

Frequency spread of the photons emitted at a zero angle $\theta=0$, as follows from the formula for $z(\omega, \theta)$, is $|\Delta \omega|=\omega_{r} \cdot \sqrt{2 / n}$. For the number of photons, generated in the sinusoidal CU with the amplitude $A$, spatial period $l$, plasma wavelength in the medium $\lambda_{p}$, one obtains the following simple formula

$$
N_{p h}=\frac{\pi \alpha \sqrt{2 n}}{2}\left(\frac{\beta_{\perp} l}{\lambda_{p}}\right)^{2}=\pi \alpha \sqrt{2 n} \cdot \eta^{2}, \quad \eta=\sqrt{2} \frac{\pi A}{\lambda_{p}},
$$

taken into account the relation $\beta_{\perp}=(2 \pi A) / l$. The parameter $\eta$ is limited by the channeling condition $\beta_{\perp}<\Theta_{L}$, which imposes a condition on the amplitude

$$
A<A_{C}, \quad A_{C}=\frac{\lambda_{p}}{2 \pi \lambda_{r}} \sqrt{2 v \lambda_{p} \lambda_{r}},
$$

where $\lambda_{r}$ is a resonant wavelength of the photon. It should be noted that the value of $A_{C}$ is weakly dependent on the choice of the crystal.

\section{Discussions and Calculations for Diamond}

Let positrons of the bunch fall at a zero angle into the sinusoidally curved planes of a crystal. Then the maximum angle of deviation of positrons from crystallographic planes is $\beta_{\perp}=(2 \pi A) / l$. Provided that $\beta_{\perp}<\Theta_{L}$, the channeling phenomenon will not be disturbed. Let estimate the parameter $q_{c u}=\gamma_{t h} \beta_{\perp}$, when the resonant wavelength $\lambda_{r}$ is from the water window frequency range $2.3 \leq \lambda_{r} \leq 4.4 \mathrm{~nm}$. Taking into account that $\gamma_{t h}=\lambda_{p} /\left(\lambda_{r} \sqrt{Q}\right)$ for $q_{c u}$, one obtains the constraint $q_{c u}<\gamma_{t h} \Theta_{L}=\sqrt{2 v \lambda_{p} \sqrt{Q} / \lambda_{r}}$. Since $\lambda_{p} / \lambda_{r} \approx 10, v \leq 10^{-4}, Q \geq 1$, then $q_{c u}<5 \cdot 10^{-2}$. Consequently, the radiation has a dipole character $Q=1$. The contribution of higher harmonics to radiation is negligible.

Let consider CU consisting of diamond crystal with sinusoidally curved $\left(\begin{array}{lll}1 & 1 & 0\end{array}\right)$ planes with amplitude $A$ and spatial period $l$. For the depth of the potential well of the average parabolic potential of the planar channel, we will use the value $U_{0}=23 \mathrm{eV}[6]\left(v=3.75 \times 10^{-5}\right)$, and for the plasma wavelength of the medium the value $\lambda_{p}=3.2 \times 10^{-6} \mathrm{~cm} \quad\left(\hbar \omega_{p}=38.8 \mathrm{eV}\right.$ [23], where $\hbar=h /(2 \pi), h$ is Planck's constant).

Let the positron bunch with energy $5 \mathrm{MeV}(\gamma=9.785)$ has the following parameters - the positron number in a bunch is $N_{b}=1.56 \times 10^{9}$, the transverse section is $S=1.18 \times 10^{-6} \mathrm{~cm}^{2}$ $\left(\sigma_{r}=6.12 \times 10^{-4} \mathrm{~cm}\right)$. We believe that it is possible to obtain a positron bunch with the same parameters as the LCLS electron bunch.

To generate a directed photon beam with the wavelength $\lambda_{r}=2.56 \mathrm{~nm}$ (with the energy of $0.484 \mathrm{keV}$ ), it is necessary to use CU with $l=4 \times 10^{-5}$ and $A=1.3 \AA$. Restriction (8) for the amplitude is taken into account, since $A_{C}=1.56 \AA$. We will assume that the dechannelling length is $0.2 \mathrm{~cm}$. Hence, the number of positron oscillations in curved planar channels CU is $n=5 \times 10^{3}$.

The number of photons emitted by a single positron of the bunch, calculated by the formula (7), is equal to $N_{p h}=7.56 \times 10^{-4}$, and the number of photons emitted by a positron bunch is 
$N_{\text {tot }}=N_{b} \times N_{p h}=1.18 \times 10^{6}$. The density of photons directed at a zero angle with the energy of $0.484 \mathrm{keV}$ and the energy spread of $2 \times 10^{-2}$ is equal to $N_{\text {tot }} / S=10^{12}$.

\section{Conclusion}

It is shown that $\mathrm{CU}$ with chosen parameters can serve as a compact emitter for generation of the soft X-ray photons, using a relativistic positron bunch. The value of the article lies in the fact that the number of emitted photons does not depend on the bunch's energy. It is possible in two cases - at the bunch's energies equal to the threshold and, in the case, when it is much higher than threshold energy. The first case is more effective, since low energy relativistic positron bunch energy is more realistic. A specific example shows the possibility of generating a monochromatic intense and directed beam of photons with the energy in the water window region. Such beams of photons are required for biological research and for practical applications in medicine.

\section{References}

[1] V.L. Ginzburg, Izvestia AN SSSR, Physica 11 (1947) 165.

[2] N.A. Korhmazyan, Izvestia AN Arm SSR, Physica 5 (1970) 287.

[3] M.A. Kumakhov, Phys. Lett. 57 (1976) 17.

[4] J. Lindhard, Kongel. Dan. Vidensk. Selsk., Mat.-Fys. Medd, 34 (1965) 30.

[5] L.A. Gevorgyan, N.A. Korkhmazyan, JETP 76 (1979) 1226.

[6] V.A. Bazilev, N.K. Zhevago, Radiation of Fast Particles in Matter and External Fields, Moscow, Nauka, 1987.

[7] V.V. Kaplin, S.V. Plotnikov, S.A. Vorobev, Zh. Tekh. Fiz. 50 (1980) 1079.

[8] R.O. Avakian, L.A. Gevorgian, K.A. Ispirian, R.K. Ispirian, NIM B 173 (2001) 112.

[9] A.A. Korol, A.V. Solov'yov, W. Greiner, J. Phys. G 24 (1998) L45.

[10] M.L. Ter-Mikaelian, High-Energy Electromagnetic Processes in Condensed Media, New York, Wiley-Interscience, 1972.

[11] E.N. Tsyganov, Fermilab preprint TM-682; TM-684 (1976).

[12] L.A. Gevorgian, K.K. Ispirian, A.H. Shamamian, NIM B 309 (2013) 63.

[13] B.T. Baranov et al., Pisma Zh. Eksp. Teor. Fiz. 82 (2005) 638.

[14] H. Backe et al., II Nuovo Cimento 34C (2010) 157.

[15] L.A. Gevorgian, N.A. Korkhmazian, Preprint EFI-272(66)-77, Erevan Physics Institute, Erevan (1977).

[16] L.A. Gevorgian, N.A. Korkhmazian, Phys. Lett. A 75 (1979) 453.

[17] L.A. Gevorgian, N.A. Korkhmazian, Patent No 784729 (1980).

[18] L.A. Gevorgian, P.M. Pogosyan, Izvestia AN Arm SSR, Fiz. 19 (1984) 239.

[19] L.A. Gevorgian, Abstracts of $17^{\text {th }}$ Intern. Free Electron Laser Conf., New York, 1995.

[20] L.A. Gevorgian, Proc. of SPIE 5974 (2005) 5974OV.

[21] A.R. Maier et al., Scientific Reports 10 (2020) 5634.

[22] K.L. Gevorgyan, L.A. Gevorgyan, Arm. J. Phys. 11 (2018) 125.

[23] R.O. Avakian, L.A. Gevorgian, K.A. Ispirian, R.K. Ispirian, JETP Letters 68 (1998) 467. 